Study Protocol

\title{
Effectiveness of a multicomponent school based intervention to reduce bullying among adolescents in Chandigarh, North India: A quasi-experimental study protocol
}

\author{
Monica Rana, ${ }^{1}$ Madhu Gupta, ${ }^{1}$ Prahbhjot Malhi, ${ }^{2}$ Sandeep Grover, ${ }^{3}$ Manmeet Kaur ${ }^{1}$ \\ ${ }^{1}$ Department of Community Medicine, School of Public Health, ${ }^{2}$ Department of Paediatrics, ${ }^{3}$ Department of \\ Psychiatry, Post Graduate Institute of Medical Education and Research, Chandigarh, India
}

Significance for public health

Bullying is one of the important but neglected health concern among adolescents especially in low and middle income countries. The results of this study might guide the policy makers and public health professionals, not only in India but in other low and middle income countries, in formulating policy or guidelines for universal implementation of bullying prevention program in the schools with an aim to improve the overall health of the adolescents, and ultimately contribute in achieving the United Nation's Sustainable Development Goals, three and four, of ensuring good health and well-being, and providing quality education, respectively.

\section{Abstract}

Background: Bullying perpetration and victimization is associated with significant academic, psychosocial and health related problems among adolescents. There is a need to develop effective interventions to prevent bullying among adolescents, especially in low and middle income countries. This paper presents the study protocol to develop, and evaluate the effect of multi-component school based prevention program for bullying in India.

Design: Quasi-experimental study.

Methods: The study will be conducted among 846 students of grade $7^{\text {th }}$ and $8^{\text {th }}$ in the intervention and control schools in Chandigarh, Union Territory, North India. A government and a private school will be selected purposively in each of the intervention and control arm. The intervention is based on socio-ecological model, and will be administered at individual, relationship (parents and teachers) and school level. The primary study outcome will be the proportion of students experiencing any kind of bullying (bullying, victimization, or both), in each study arm. The effectiveness of the intervention will be measured by performing difference in difference analysis and generalized estimating equations.

Expected impact for public health: Bullying is an aggressive behaviour with significant morbidities, including psychological or physical trauma, affecting individuals not only in their adolescence, but also later in their adulthood. This quasi-experimental study is expected to provide evidence on whether multi-component bullying prevention intervention program, can reduce the burden of bullying perpetration and victimization among school adolescents in India. The results of the study will add in the exiting literature on bullying intervention program, especially, from the low middle-income countries, as there are limited studies available on this topic in these countries.

\section{Introduction}

Bullying is an aggressive behaviour with significant morbidities affecting individuals not only in their adolescence, but also later in their adulthood. ${ }^{1}$ It is a common stressful experience affecting on an average 35\% adolescents worldwide, especially, during school hours. ${ }^{2}$ Bullying behaviour is recognized by an imbalance of power, repetitiveness, and the intention of bully to harm the victim. ${ }^{3}$ Bullying can range from less serious situations, like teasing or name calling, to very serious physical, sexual or verbal abuse, which can result into psychological or physical trauma, which may continue till adulthood. ${ }^{4}$ Bullying behaviours have emerged as a significant factor influencing the adjustment of both bullies and victims and the learning environment of all the students. It is reported that adolescent's role as a perpetrator, victim, or bystander, is associated with significant academic, psychosocial and health related problems. One highly worrisome outcome that has been found to be particularly high among bullies and victims is suicide. ${ }^{5}$

A study from India, reported the prevalence of aggression among students studying in grades $9^{\text {th }}$ to $10^{\text {th }}$ to be $53 \%(19.2 \%$ victims and $13 \%$ bullies). ${ }^{6}$ This indicates that problem of bullying exists in India. Studies that focus on bullying in India are more important because the education system is India is highly achievement oriented, which involves long hours of reading and memorizing lessons. This puts the students with additional mental stress, anxiety and frustration, which is a precipitator of bullying. ${ }^{7}$ Educational system in India is divided into government and private schools. There is no documented difference between government and private schools prevalence in regard to bullying. However, in government schools, generally students from lower/middle socioeconomic status are enrolled, and in private schools, students from all (low/middle/high) socioeconomic status are enrolled. In our earlier unpublished study (Rana et al.), ${ }^{8}$ it is observed that prevalence of any kind of bulling is higher among students studying in the private schools $(33.1 \%)$ as compared to the students in the government school (19.2\%). Also type of school (government vs private) is a significant predictor of bullying $(\mathrm{OR}=0.63)$. Thus, it is important to plan and conduct a bullying prevention intervention studies in India. Additionally, such studies will add to the limited literature available regarding development or impact of bullying prevention interventional program per se in schools in India, which has the world's second largest adolescent's population. ${ }^{9}$ To deal with bullying, school based anti-bullying prevention programs have been developed and evaluated in the high income countries (HICs). Though their curricula vary but in general, these provide students with information about bullying, 
intend to change how adolescent think and feel about it, and develop adolescent's skills to avoid bullying and resolve disputes through nonviolent means. Olweus bullying prevention program was one of the first intervention program to target bullying problem in schools in Norway. ${ }^{10}$ It was successful in significantly reducing victimization (10\% to $3.6 \%)$ and perpetration among the school students ( 7.6 to $3.6 \%$ ). A systematic review of school based antibullying intervention program reported that the whole school based interventions reduce bullying perpetration and victimization by an average of $17-23 \%$. It is also reported that interventional programs that are of shorter duration, with no parental involvement or that lack whole school approach, have less effect on bullying behaviours. ${ }^{11}$ The antibullying intervention in this study is developed using the whole school based approach along with involvement of parents, so as to overcome the limitations of the previous intervention programs. Lancet's commission on Adolescent Health and Well Being has pointed towards the need of interventions to improve adolescent health in Lower Middle Income Countries (LMICs), where focus from several decades has been on reproductive and sexual health, and important health priorities like mental heath, violence and injuries have been neglected. ${ }^{12}$ LMICs are the countries that has gross national income per capita between $\$ 1006$ and $\$ 3955$, as per World Bank classification of countries based upon income. ${ }^{13}$ These efforts are expected to contribute in achieving the United Nation's Sustainable Development Goals, three and four, of ensuring good health and well-being, and providing quality education, respectively. ${ }^{14}$

In view of this, this study is planned with the objective to first design and develop a multi-component prevention program on bullying perpetration and victimization, and then examine the effectiveness of this intervention in reducing the bullying perpetration and victimization among school adolescents in Chandigarh, a North Indian Union Territory. We hypothesize that a multi-component school based intervention package will reduce the proportion of adolescents involved in bullying, victimization and perpetration in the post intervention phase.

\section{Materials and Methods}

\section{Study design}

The study design of this study is quasi experimental, where the groups are non-randomized into intervention and control groups, and selected purposively.

\section{Sample}

This study is conducted in the schools located in Chandigarh, Union Territory. Indian educational system is structured into two types of school: Government schools (funded by government) and private schools (not funded by government, and administered by private organizations). There are a total of 175 middle (upto $8^{\text {th }}$ grade), high (upto $10^{\text {th }}$ grade) and senior secondary (upto $12^{\text {th }}$ grade) schools in Chandigarh. Among these, 106 are government and 69 are private schools. All these schools were eligible to participate in this study, except only boys $(n=2)$ and only girls $(n=4)$ schools, so as to have equal representation from boys and girls.

We chose to conduct this intervention study among students studying in grades $7^{\text {th }}$ and $8^{\text {th }}$, because earlier studies have reported that bullying tend to peak in middle school years and early adolescence.

Only four schools are purposively selected out of 175 schools, including one government and one private school each in the inter- vention and control arm, due to manpower and time constraints. No randomization is done because full voluntary cooperation and participation of the intervention schools is required for the implementation of the interventions. The selection of the schools is based upon their willingness to participate in the study and implement the bullying intervention program (intervention arm), the strength of students (meeting the sample size requirement) in $7^{\text {th }}$ and $8^{\text {th }}$ classes, and ratio of male to female students (1.5:1, so as to represent the overall male to female student's ratio in Chandigarh's schools).

The control schools are carefully chosen so that they have similar baseline characteristics like strength of the school, location of the school, language medium of the school, as of the intervention schools. Number of sections and students studying in each section of $7^{\text {th }}$ and $8^{\text {th }}$ grade are first listed. All the sections and students in $7^{\text {th }}$ and $8^{\text {th }}$ grade of selected schools are enrolled in the study. Students who will change schools within the study period will not be followed up. This proportion is projected to be $2 \%$, by reviewing the school records of the last year.

Although all the children studying in $7^{\text {th }}$ and $8^{\text {th }}$ classes in the selected schools are enrolled in the study, yet sample size calculations is done to ensure if adequate sample is obtained to draw conclusions. The assumption in analysing school based study is that data within cluster, i.e. classes or schools, are more similar than between clusters. The clustering effect has to be taken into account, which statistically is defined as $1+(m-1) p$, where $m$ is number of units in a cluster (students within a section of a class), and $p$ is intraclass correlation coefficient (ICC)..$^{15}$ On the basis of prior research, the expected ICC is 0.02 for both victimization and perpetration as the outcome. ${ }^{16}$ On an average, a section of a class approximately comprises of 40 students, thus leading to clustering effect of 1.78. Assuming the primary outcome (proportion of children with any bullying) to be $31 \%,{ }^{17} 12 \%$ reduction in bullying after implementation of the intervention (on an average school based intervention program reported to reduce bullying by 17 $23 \%),{ }^{6}$ significance level of $5 \%$, power of $80 \%$, non response rate of $5 \%$, the sample size is estimated to be 416 students in each arm.

The total students recruited in this study are 846 (425 in control and 421 in intervention arm), which indicates that the sample size is adequate. Mean age of the students is $12.68(\mathrm{SD}=0.77)$. Males $(57 \%)$ to females ratio $(43 \%)$ is $1.4: 1$. Students from $8^{\text {th }}$ $(52 \%)$ and $7^{\text {th }}$ grade $(48 \%)$ are almost equally represented.

\section{Study period}

The study has been ongoing since October 2016. Baseline information was collected in November- December 2016. The study will be continued till January 2018.

\section{Instruments}

A baseline assessment was done, in the intervention and control schools, to measure the burden of bullying and its correlates using self-administered Revised Olweus Bully/victim Questionnaire (Olweus 1996), ${ }^{18}$ Rosenberg self esteem questionnaire and strength and difficulties questionnaire, ${ }^{19,20}$ respectively. The Olweus Bully-victim Questionnaire provides information on bullying in the past 6 months. It is a validated tool with cronbach's alpha of 0.80 . The questionnaire contains 40 items including questions on verbal, relational physical and cyber bullying. Participants will be categorized into one of the four bullying subgroups according to their frequency of responses with bullying: Not involved participants: defined as who had not been bullied/bullied others or involved only once or twice; Victim: categorized as who had been bullied 2 or 3 times a month or more; Bully: categorized who bullied other 2 or 3 times a month or more; Bully-victim: Involved in 
bullying others and had been bullied 2 or 3 times a month or more.

Self-esteem score of the students will be measured as per Rosenberg's self esteem questionnaire. This is a 10 item scales, each of which is scored on a four point scale. This scale generally has a high reliability, and is also validated on Indian population with cronbach's alpha of 0.81 . For item $1,2,4,6$, and 7 , the score of 4 indicated strongly agree and score 1 indicated strongly disagree, and for the rest of the items the scoring was reversed as strongly agree indicated by 1 and strongly disagree indicated by 4 (higher scores indicating higher self-esteem).

Social and emotional well being of children which will be measured by total difficulty scores as per the strength and difficulty questionnaire (SDQ). It is a validated tool on Indian population with good psychometric properties and is available in English as well as Hindi versions with cronbach's alpha of 0.61. There are 25 items in SDQ which comprise of 5 sub-scales: emotional problems scale, conduct problems scale, hyperactivity scale, peer problems scale and pro-social scale. Students have to respond on a three point scale not true, somewhat true or certainly true on the basis of their behaviour in last 6 months. A total difficulties behaviour score will be calculated by summing scores from all the scales except the pro social scale (higher scores indicates greater problem).

Before the data collection, the author had visited all the sections of $7^{\text {th }}$ and $8^{\text {th }}$ classes, in the intervention and control schools, to inform the children about the study, and encourage them to participate. Parental consent of the students for participation in the study was obtained through the school principal. In addition, assent from the students themselves was also obtained. The questionnaires were administered to all those adolescents who assented/consented to participate. This phase of the study was conducted from November to December, 2016.

\section{Data analysis}

Data will be entered into statistical package for social sciences, version 16.0, which will be used for all statistical purposes. The outcome measures will be measured at individual level (students level), teachers, parents and school level. The primary and secondary outcome measures are enlisted in Table 1.

Mc-Nemar chi-square test wiil be used to see significant differences between group pre- and post test. ANOVA will be used to estimate significant difference between means of groups pre- and post test. Further difference-in-differences (DID) analysis will be used to compare the changes over time between intervention and control groups to estimate effectiveness of the intervention. Hence, in this study the change in bullying prevalence in intervention group minus the change in bullying prevalence in control group is estimated by DID. DID remove the initial difference in control and intervention group, which is not removed by single difference and hence does not underestimate the program impact. Further logistic mixed model analysis will be done to calculate relative risks in control schools as compared to intervention schools. Differences between groups will be considered significant at $95 \%$ confidence interval. To analyze the longitudinal differences (pre- and post intervention) in categorical variables, generalized estimating equation (GEE) method across three timelines. Repeated measures ANOVA will be used to assess the impact of intervention on the means of scores of correlates of bullying (difficulty score and self esteem score).

\section{Designing and pretesting phase of the intervention program}

The intervention program is designed and finalized after indepth review of previously published literature on bullying (Supplementary Table 1), effectiveness of anti-bullying intervention programs; conducting focus group discussions with parents, teachers and adolescents (to explore the perceptions, beliefs and suggestions regarding bullying prevention program) and conducting a consultation workshop with all the stakeholders (teachers, principals, school counsellors, students, psychiatrists and public health experts). The bullying intervention program was pretested among 60 adolescents studying in $7^{\text {th }}(n=30)$ and $8^{\text {th }}(n=30)$ grades in a school located outside the study area for feasibility, applicability of the content, and estimating the duration for delivery of each component of the intervention program per school. Pretesting was done in October 2016.

\section{Intervention phase}

Intervention in this study is based on Social Ecological Model, ${ }^{21}$ as it incorporates all the factors affecting adolescent's behaviours. The key concept of this model is that it uses individual level, relationship level and community level factors to understand the factors that influence the adolescent's behaviour. The prevention strategies that work at all levels of the social-ecological model are necessary for deep and sustained prevention of bullying

Table 1. Outcome measures to assess the effectiveness of bullying prevention program.

\begin{tabular}{|c|c|}
\hline Primary outcome measure & $\begin{array}{l}\text { Proportion of students who are involved twice or more in any kind of bullying } \\
\text { (including victimization, bullying, bully-victim) }\end{array}$ \\
\hline $\begin{array}{l}\text { Secondary outcome measure, } \\
\text { individual level }\end{array}$ & $\begin{array}{l}\text { Proportion of students who are involved twice or more as bullies only. Proportion of students who are involved twice or } \\
\text { more as victims only. Proportion of students who are involved twice or more as bully-victims only. } \\
\text { Proportion of students who felt that other students try to stop bullying in school. } \\
\text { Proportion of students who felt sorry or helped the victim student to stop bullying. } \\
\text { Proportion of students who feel that students deserve to be bullied. }\end{array}$ \\
\hline $\begin{array}{l}\text { Secondary outcome measure, } \\
\text { correlates of bullying }\end{array}$ & $\begin{array}{l}\text { Mean self esteem score (Rosenberg self esteem scale). } \\
\text { Mean difficulties score (Strength and difficulty questionnaire). }\end{array}$ \\
\hline $\begin{array}{l}\text { Secondary outcome measure, } \\
\text { teacher or parents level }\end{array}$ & $\begin{array}{l}\text { Proportion of students who felt teachers or adult try to stop bullying in the school. } \\
\text { Proportion of victims, whose parents contacted school to stop bullying. } \\
\text { Proportion of students who felt their class teachers has done a good deal or more to counteract the bullying in class. } \\
\text { Proportion of bullies who were communicated by teacher or adult in school to stop them from bullying other children. } \\
\text { Proportion of bullied who were communicated by their parents to stop them from bullying other children. }\end{array}$ \\
\hline $\begin{array}{l}\text { Secondary outcome measure, } \\
\text { school level }\end{array}$ & $\begin{array}{l}\text { Proportion of students who feel safe from getting bullied in school. } \\
\text { Proportion of times an action is taken by the school committee to prevent bullying in the school }\end{array}$ \\
\hline
\end{tabular}


behaviour. Therefore, the intervention strategies are first formulated at these three levels to prevent bullying in this study, as given in Figure 1.

The multi-component school based intervention strategy will be administered at three levels i.e., individual (students), relationship (parents and teachers), and school level. Details of the intervention package are provided in the Supplementary Table 2. Brief description of the package is given below:

\section{Individual level strategy (classroom-based curriculum)}

The classroom-based curriculum will be delivered through conducting group meetings with the adolescents in their classrooms by the authors themselves. The group meetings will be facilitated by the power point presentations, videos, and structured group activities, so as to maintain the homogeneity in the content of intervention in each school. Videos and group activities will make the session interactive and interesting for the students, and aim at enhancing the participation of the students in the intervention. Three group activities will be conducted including: i) slogan making competition on bullying; ii) poster making competitions depicting the problem of bullying in schools; and iii) experience sharing sessions on bullying incidents in school. The activities will be conducted in groups and will be conducted after the powerpoint sessions. The group activities will help in understanding whether students developed the understanding of the sessions. A separate talk on cyber bullying, and the ways to prevent it will also be given to all the enrolled students. The content of the intervention will be bilingual (in Hindi and English language). This classroom curriculum will be delivered in three sessions. The duration of two sessions will be one-hour each, and third session will be of 2 hours duration. The classroom-based curriculum is aimed at increasing their knowledge regarding bullying behavior and its harmful effects and strengthening their skills to prevent bullying victimization.

\section{Relational level strategy (parents and teachers)}

Relationship level strategy will be delivered through parent and teachers module separately.

Parent's module will be delivered by conducting two group meetings with the parents of one hour duration each. These meetings will be organized on the parent-teacher-meeting day in the school premises. Parents will be first oriented about bullying problem, and then explained about the signs of bully or victim so that they can identify if their child is a bully or a victim or has been witnessing bullying in school. Specific parenting skills will be imparted to the parents to enable them to tackle bullying if their child has been involved in it. A talk on cyber bullying and its preventive strategies will also be delivered to parents. These meetings will be facilitated by power-point presentations in the bilingual language. In addition, on the subsequent parent-teacher-meeting (once every month) days, which will be held during the intervention phase, an interactive session will be held with the parents to clarify and discuss their doubts.

In the teacher's module, classroom rules and teaching skills will be provided to the teachers in the group meetings with them, to prevent bullying in the class or school. Teachers will be provided with the ways (To do list) to respond to the bullying, like talking to bully and victim separately to understand the situation, and most importantly is to tell the victim not to ignore the bullying. A session on relationship building with adolescents will be held, as it is an important strategy for preventing adolescents to involve in bullying. This content will be delivered in both Hindi and English depending upon the medium of the language used in the school, and will be facilitated by power point presentation. There will be 2 sessions of one hour duration each with the teachers. Teachers will be followed up every week, to make sure they are implementing the intervention program.

\section{Community level strategy (school level)}

Community level strategy will be delivered by creating enabling environment in the school and sensitizing the school administrators regarding the bullying problem. Conducting group meetings with the school administrators including school principal, vice-principal and school coordinators will do this. A committee comprising of principal, vice principal, counselor and two teachers will be formulated to deal with the bullying problem and implement bullying prevention program in the intervention schools. A meeting with the committee once every month will be held to ensure proper implementation of the program. This committee will also introduce school rules against bullying, and display messages and posters against bullying on bulletin boards with in the school premises.

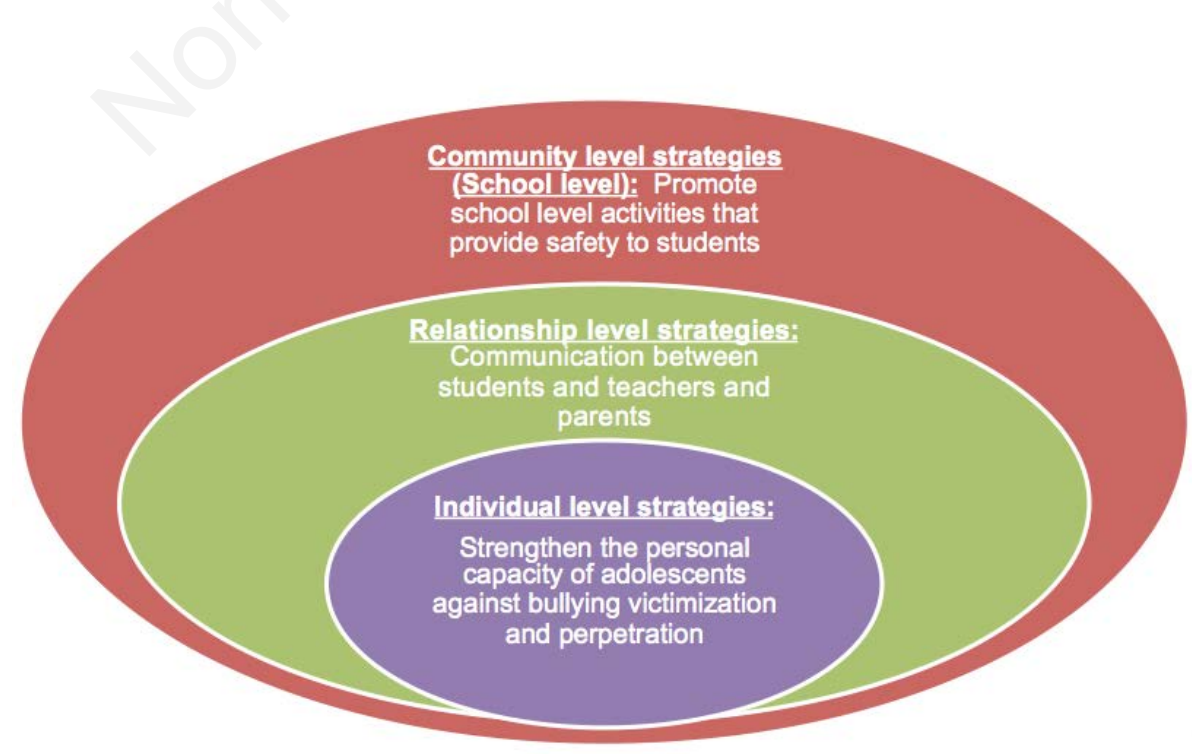

Figure 1. Social ecological model based strategies to prevent bullying. 
The extent of implementation of the intervention program will be measured by assessing the status of various program components twice in a month during the intervention phase.

\section{Control schools}

In the control schools, the school authorities will conduct the routine adolescent health sessions like reproductive and sexual health.

\section{Post intervention phase (endline assessment)}

Mid- and end-line assessment will be done at three and eight months, respectively, of implementing the intervention on the same students using the same questionnaire, to measure bullying and its correlates, as used in the baseline assessment of the study.

\section{Ethical consideration}

Ethics committee of Postgraduate Institute of Medical Education and Research has approved this study (Approval no: INT/IEC/2015/258). Prior permission to conduct the study in schools has been obtained from Director Public Instructions (DPI), Chandigarh. Written consent from the parents of the adolescents and assent from adolescents in the language they understand is obtained before conducting the study. The respondents were briefed about the purpose and objective of the study by providing the study information sheet. The information collected from the students will be kept confidential and anonymous. The study will be contributing to $\mathrm{PhD}$ public health thesis for the first author.

\section{Discussion}

This quasi-experimental study is expected to show whether multi-component bullying prevention intervention program will contribute in reducing the burden of bullying perpetration and victimization among adolescent students. The present intervention program has been designed considering the needs of the stakeholders (students, parents and school staff) by conducting focus group discussions, which is essential for the success of any intervention. The effectiveness of the program will be evaluated by collecting post intervention data twice which will give short as well as long term effects of the program.

Bullying is a significant public health problem because it has serious long term harmful health consequences. Due to the harmful consequences of bullying, several studies have been conducted to evaluate the effectiveness of anti-bullying prevention programs. With one in every three children ${ }^{2}$ involved in some form of bullying, ${ }^{2}$ it is important to have effective antibullying interventions as bullying is accompanied with devastating consequences. Even though violence and bullying are higher in less developed countries, majority of the intervention studies reported are from European and North American countries. ${ }^{22}$ Therefore, the present study will contribute to new scientific evidence on effectiveness of bullying prevention programs in LMICs, where there is very less research on this topic.

The strength of this study is that it is a whole school based intervention program that involves not only students but school administrators, teachers and parents, who play an important role in the success of any school program for adolescents. The use of videos will also be done for increasing the interest of the adolescents on this issue. Regular follow up with the school administrators and teachers will be done for increasing the extent of the implementation of the intervention. The present intervention program focuses on both traditional and cyberbullying. The quasi-experimental study design was purposefully chosen for this study, because motivation and full co-operation of the school administration is required for the successful implementation of the program in the schools. Previous intervention programs have also documented that quasi experimental study design has been one of the reasons for the success of bullying programs. Zych et al., ${ }^{23}$ reviewed 10 anti-bullying intervention programs and concluded successful anti-bullying intervention program components like antibullying committee, rules against bullying, improving school environment and involving families. These components have been included in the present bullying intervention program. However, this design has limitations like lack of randomization which results into selection bias both at individual and school level. To minimize the bias baseline characteristics of the study population were matched. However, potential sources of selection bias like socioeconomic status of students, strength of school can be included as confounders in the analysis to reduce the effect of selection bias in the estimates. Another limitation of the study is the use of self administered questionnaire in which students may find difficulty in understanding few questions. To tackle this limitation, the authors had explained all the questions to the participants before administering the questionnaire.

The public health implication of this study is that it will generate evidence on the effectiveness of the intervention in dealing with bullying problem among students, which can guide the policy makers on formulating policy or guidelines for universal implementation of this program in the schools not only in India, but in other low and middle income countries as well.

\section{Trial registration}

The study protocol is registered with Clinical Trials Registry, India. Registration number is CTRI/2017/04/008413.

Correspondence: Madhu Gupta, Department of Community Medicine and School of Public Health, Room No. 130, PGIMER, Chandigarh, India. Tel.: +91.1722690727 - Fax: +911722744401.

E-mail: madhugupta21@gmail.com

Key words: Adolescents; Bullying; Intervention; Schoolbased; Victimization. Acknowledgements: the authors would like to thank Director Public Instructions, Education department Chandigarh for granting permissions to conduct the study.

Contributions: MR developed the first draft of the manuscript. MG, SG, PM and MK contributed to the conception and design of the study. All authors provided feedback during manuscript development. Each author has read and approved the final manuscript.

Conflict of interest: the authors declare no potential conflicts of interest. Funding: MR is funded by Indian Council of Medical Research, New DelhiJunior Research Fellowship (JRF) for undergoing PhD program.

Received for publication: 9 November 2017.

Revision received: 15 March 2018.

Accepted for publication: 13 April 2018.

CC Copyright M. Rana et al., 2018

Licensee PAGEPress, Italy

Journal of Public Health Research 2018;7:1304

doi:10.4081/jphr.2018.1304

This work is licensed under a Creative Commons Attribution NonCommercial 4.0 License (CC BY-NC 4.0). 


\section{References}

1. Srabstein J, Leventhal B. Prevention of bullying related morbidity and mortality: a call for public health policies. Bull WHO 2010;88:403.

2. Modecki K, Minchin J, Harbaugh A, et al. Bullying prevalence across contexts: A meta-analysis measuring cyber and traditional bullying. J Adolesct Health 2014;55:602-11.

3. Olweus D. Bullying at school: What we know and what we can do. Oxford \& Cambridge: Blackwell Publishers; 1993.

4. Klomek A, Sourander A, Elonheimo H. Bullying by peers in childhood and effects on psychopathology, suicidality and criminality in adulthood. Lancet Psychiatry 2015;2:930-41.

5. Kelly EV, Newton NC, Stapinski LA, et al. Suicidality, internalizing problems and externalizing problems among adolescent bullies, victims and bully victims. Prev Med 2015;73:1005.

6. Malhi P, Bharti B, Sidhu M. Aggression in schools: psychosocial outcomes of bullying among Indian adolescents. Indian $\mathrm{J}$ Pediatr 2014;81:1171-6.

7. Deb S, Strodl E, Sun J. Academic Stress, Parental Pressure, Anxiety and Mental Health among Indian High School Students. Int J Psychol Behav Sci 2015;5:26-34.

8. Rana M, Gupta M, Malhi P, et al. Prevalence and correlates of bullying perpetration and victimization among adolescents in schools in Chandigarh; 2018.

9. United Nations Children's Fund (UNICEF). The state of the World's children 2011: Adolescence: An age of opportunity. February 2011. New York USA.

10. Smith PK, Morita Y, Junger-Tas J, et al. The nature of school bullying: A cross-national perspective. London: Routledge; 1998. pp 28-48.

11. Ttofi M, Farrington D. Effectiveness of a school based programs to reduce bullying: a systematic and meta-analytic review. J Exp Criminol 2011;7:27-56.
12. Patton G, Sawyer S, Santelli J, et al. Our future: Lancet commission on adolescent health and well being. Lancet 2016;387:2423-78.

13. The World Bank data. Lower middle income countries. Available from: https://data.worldbank.org/incomelevel/lower-middle-income. Accessed on 11 March 2018.

14. UNPD. Sustainable development goal. Available from: http://www.undp.org/content/undp/en/home/sustainabledevelopment-goals.html. Accessed on 11 March 2018.

15. Hemming K, Girling A, Sitch A, et al. Sample size calculation for cluster randomized controlled trials with fixed number of clusters. BMC Medical Research Methodol 2011;11:102.

16. Karna A, Voeten M, Little TD, et al. A large scale evaluation of the KiVa antibullying program: grades 4-6. Child Deve 2011;82:311-30.

17. Kshirnagar VY, Agarwal R, Bavdekar SB. Bullying in Schools: Prevalence and short-term Impact. Indian Paediatrics 2007;44:25-27.

18. Olweus D. The revised Olweus Bully/Victim Questionnaire. Bergen: Research centre for health promotion; 1996.

19. Blascovich J, Tomaka J. Measures of self-esteem. In: Robinson J, Shaver P, Wrightsman L, eds. Measures of Personality and Social Psychological Attitudes. 3 rd ed. Ann Arbor: Institute for Social Research; 1993. pp. 115-160.

20. Goodman R. The strengths and difficulties questionnaire: A research note. J Child Psychol Psychiatry 1997;38:581-6.

21. Bronfenbrebber U. The ecology of human development: Experiments by nature and design. Cambridge, Mass: Harvard University Press; 1979.

22. Zych I, Ortega-Ruiz R, Rey RD. Scientific research on bullying and cyberbullying: where have we been and where are we going. Aggression Violent Behav 2015;24:188-98.

23. Zych I, Farrington DP, Llorent VJ, Ttofi MM. Protecting Children against bullying and its consequences. Switzerland: Springer; 2017. 